\title{
Measurement of the Neutron Induced Fission Cross Section on Transuranic (TRU) Elements at the n_TOF Facility at CERN
}

Cite as: AIP Conference Proceedings 947, 43 (2007); https://

doi.org/10.1063/1.2813848

Published Online: 31 October 2007

P. F. Mastinu, U. Abbondanno, G. Aerts, H. Álvarez, F. Álvarez-Velarde, S.

Andriamonje, J. Andrzejewski, P. Assimakopoulos, L. Audouin, G. Badurek, P.

Baumann, F. Becvár, E. Berthoumieux, F. Calviño, M. Calviani, D. Cano-Ott, R. Capote, C. Carrapiço, P. Cennini, V. Chepel, E. Chiaveri, N. Colonna, G. Cortes, A. Couture, J. Cox, M. Dahlfors, S. David, I. Dillman, C. Domingo-Pardo, W. Dridi, I. Duran, C. Eleftheriadis, M. Embid-Segura, L. Ferrant, A. Ferrari, R. Ferreira-Marques, K. Fujii, W. Furman, I. Goncalves, E. González-Romero, F. Gramegna, C. Guerrero, F. Gunsing, B. Haas, R. Haight, M. Heil, A. Herrera-Martinez, M. Igashira, E. Jericha, F. Käppeler, Y. Kadi, D. Karadimos, D. Karamanis, M. Kerveno, P. Koehler, E. Kossionides, M. Krticka, C. Lampoudis, H. Leeb, A. Lindote, I. Lopes, M. Lozano, S. Lukic, J. Marganiec, S. Marrone, T. Martínez, C. Massimi, A. Mengoni, P.M. Milazzo, C. Moreau, M. Mosconi, F. Neves, H. Oberhummer, S. O'Brien, J. Pancin, C. Papachristodoulou, C. Papadopoulos, C. Paradela, N. Patronis, A. Pavlik, P. Pavlopoulos, L. Perrot, M. T. Pigni, R. Plag, A. Plompen, A. Plukis, A. Poch, J. Praena, C. Pretel, J. Quesada, T. Rauscher, R. Reifarth, C. Rubbia, G. Rudolf, P. Rullhusen, J. Salgado, C. Santos, L. Sarchiapone, I. Savvidis, C. Stephan, G. Tagliente, J. L. Tain, L. Tassan-Got, L. Tavora, R. Terlizzi, G. Vannini, P. Vaz, A. Ventura, D. Villamarin, M. C. Vincente, V. Vlachoudis, R. Vlastou, F. Voss, S. Walter, M. Wiescher, and K. Wisshak

\section{ARTICLES YOU MAY BE INTERESTED IN}

Study of Neutron-Induced Fission Cross Sections of U, Am, and Cm at n_TOF AIP Conference Proceedings 1265, 477 (2010); https:// doi.org/10.1063/1.3480244

Measurement of ${ }^{139} \mathrm{La}(\mathrm{n}, \gamma)$ Cross Section at $\mathrm{n}_{-}$TOF AIP Conference Proceedings 831, 551 (2006); https:// doi.org/10.1063/1.2201012 
AIP Conference Proceedings 831, 502 (2006); https://

doi.org/10.1063/1.2200996

\section{Lock-in Amplifiers up to $600 \mathrm{MHz}$}
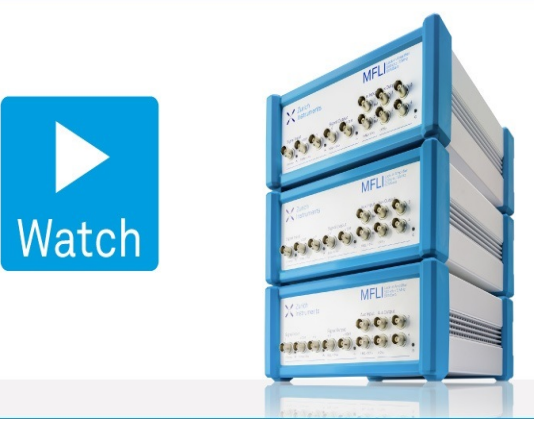

AIP Conference Proceedings 947, 43 (2007); https://doi.org/10.1063/1.2813848 


\section{Measurement of the Neutron Induced Fission Cross Section on Transuranic (TRU) Elements at the n_TOF Facility at CERN}

P. F. Mastinu ${ }^{12}$, U. Abbondanno ${ }^{1}$, G. Aerts ${ }^{2}$, H. Álvarez ${ }^{3}$, F. Álvarez-Velarde ${ }^{4}$, S. Andriamonje ${ }^{2}$, J. Andrzejewski ${ }^{5}$, P. Assimakopoulos ${ }^{\dagger}{ }^{6}$, L. Audouin ${ }^{7}$, G. Badurek ${ }^{8}$, P. Baumann ${ }^{9}$, F. Becvár ${ }^{10}$, E. Berthoumieux ${ }^{2}$, F. Calviño ${ }^{11}$, M. Calviani ${ }^{38}$, D. Cano-Ott ${ }^{4}$, R. Capote ${ }^{13,14}$, C. Carrapiço ${ }^{15,2}$, P. Cennini ${ }^{16}$, V. Chepel ${ }^{17}$, E. Chiaveri ${ }^{16}$, N. Colonna ${ }^{18}$, G. Cortes ${ }^{19}$, A. Couture ${ }^{20}$, J. Cox ${ }^{20}$, M. Dahlfors ${ }^{16}$, S. David ${ }^{7}$, I. Dillman ${ }^{21}$, C. Domingo-Pardo ${ }^{22}$, W. Dridi ${ }^{2}$, I. Duran ${ }^{3}$, C. Eleftheriadis ${ }^{23}$, M. Embid-Segura ${ }^{4}$, L. Ferrant ${ }^{\dagger 7}$, A. Ferrari ${ }^{16}$, R. FerreiraMarques $^{17}$, K. Fujii ${ }^{1}$, W. Furman ${ }^{24}$, I. Goncalves ${ }^{17}$, E. González-Romero ${ }^{4}$,

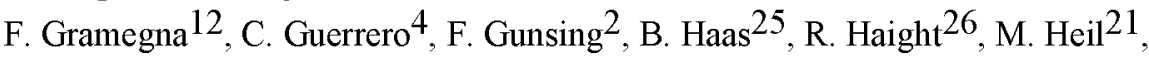
A. Herrera-Martinez ${ }^{16}$, M. Igashira ${ }^{27}$, E. Jericha ${ }^{8}$, F. Käppeler ${ }^{21}$, Y. Kadi ${ }^{16}$, D. Karadimos ${ }^{6}$, D. Karamanis ${ }^{6}$, M. Kerveno 9 , P. Koehler ${ }^{28}$, E. Kossionides ${ }^{29}$, M. Krticka ${ }^{10}$, C. Lampoudis ${ }^{23,2}$, H. Leeb $^{8}$, A. Lindote ${ }^{17}$, I. Lopes ${ }^{17}$, M. Lozano ${ }^{14}$, S. Lukic ${ }^{9}$, J. Marganiec ${ }^{5}$, S. Marrone ${ }^{18}$, T. Martínez ${ }^{4}$, C. Massimi ${ }^{30}$, A. Mengoni ${ }^{13,16}$, P.M. Milazzo ${ }^{1}$, C. Moreau ${ }^{1}$, M. Mosconi ${ }^{21}$, F. Neves ${ }^{17}$, H. Oberhummer ${ }^{8}$, S. O'Brien ${ }^{20}$, J. Pancin ${ }^{2}$, C. Papachristodoulou ${ }^{6}$, C. Papadopoulos ${ }^{31}$, C. Paradela ${ }^{3}$, N. Patronis ${ }^{6}$, A. Pavlik ${ }^{32}$, P. Pavlopoulos ${ }^{33}$,

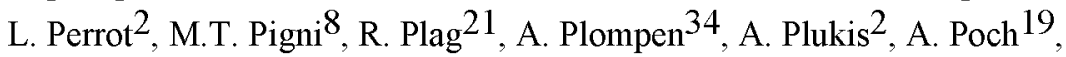
J. Praena ${ }^{12}$, C. Pretel ${ }^{19}$, J. Quesada ${ }^{14}$, T. Rauscher ${ }^{35}$, R. Reifarth ${ }^{26}$, C. Rubbia ${ }^{36}$, G. Rudolf ${ }^{9}$, P. Rullhusen ${ }^{34}$, J. Salgado ${ }^{15}$, C. Santos ${ }^{15}$, L. Sarchiapone ${ }^{16}$, I. Savvidis ${ }^{23}$, C. Stephan ${ }^{7}$, G. Tagliente ${ }^{18}$, J.L. Tain ${ }^{22}$, L. Tassan-Got ${ }^{7}$, L. Tavora ${ }^{15}$, R. Terlizzi ${ }^{18}$, G. Vannini ${ }^{30}$, P. Vaz ${ }^{15}$, A. Ventura ${ }^{37}$, D. Villamarin ${ }^{4}$,

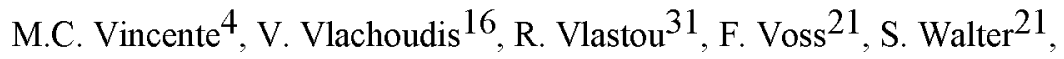
M. Wiescher ${ }^{20}$, K. Wisshak 21

${ }^{1}$ Istituto Nazionale di Fisica Nucleare, Trieste, Italy

${ }^{2}$ CEA/Saclay - DSM/DAPNIA, Gif-sur-Yvette, France

${ }^{3}$ Universidade de Santiago de Compostela, Spain

${ }^{4}$ Centro de Investigaciones Energeticas Medioambientales y Tecnologicas, Madrid, Spain

${ }^{5}$ University of Lodz, Lodz, Poland

CP947, VII Latin American Symposium on Nuclear Physics and Applications

edited by R. Alarcon, P. L. Cole, C. Djalali, and F. Umeres

(C) 2007 American Institute of Physics 978-0-7354-0461-8/07/\$23.00 


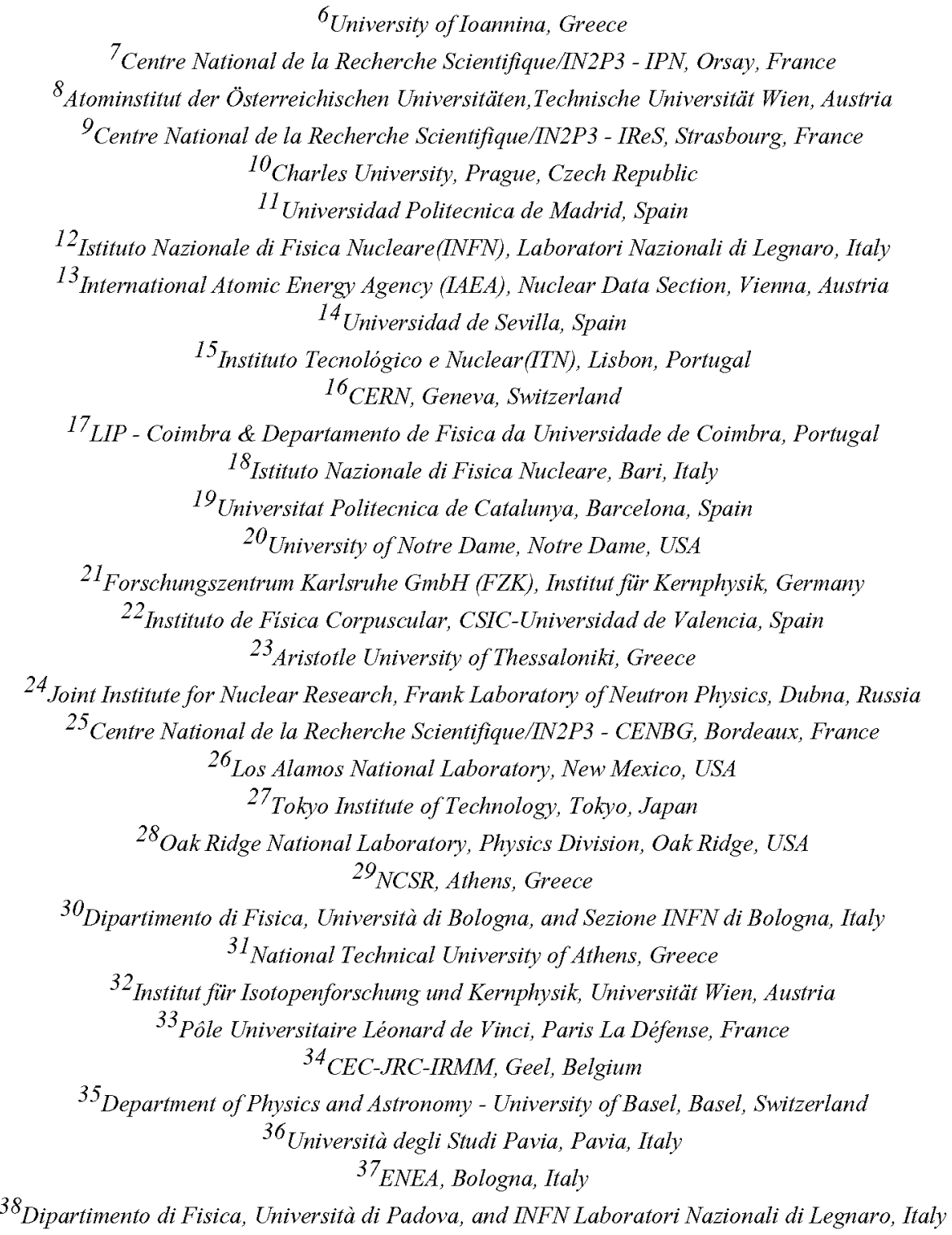

\begin{abstract}
During the 2004 campaign, the $n$ TOF collaboration measured neutron fission cross sections for ${ }^{233} \mathrm{U},{ }^{241,243} \mathrm{Am},{ }^{245} \mathrm{Cm}$, as well as the fission standards ${ }^{235,238} \mathrm{U}$, using a sealed Fission Ionization Chamber (FIC). The setup included a total of 16 targets and 18 electrodes mounted together in a $50-\mathrm{cm}$ length chamber, allowing the measurements of all isotopes at the same time, thus in the same experimental conditions. A brief description of the facility and of the detector setup will be presented followed by the preliminary results of the analysis of ${ }^{235} \mathrm{U},{ }^{233} \mathrm{U}$, and ${ }^{245} \mathrm{Cm}$ from thermal energies up to some tenths of $\mathrm{MeV}$.
\end{abstract}

PACS: $01.30 . \mathrm{Cc}+14.20 . \mathrm{Dh}+28.20 .-\mathrm{v}+25.85 .-\mathrm{w}$ 


\section{INTRODUCTION}

Recently, potential climate problems related to the greenhouse-gas effect have triggered a renewed interest in nuclear energy production, mainly because nuclear power is the only energy source that combines the attributes of large-scale electricity production, and zero greenhouse gas emissions during the production process. In addition, many countries consider nuclear energy a strategic component of a diverse energy portfolio to meet fast-growing electricity demand and decrease dependence on foreign governments/companies. However, before becoming truly competitive, nuclear energy will have to solve problems related with safe operation, and waste production during its full life cycle.

Although much progress has been made towards safer operation during the last few decades, radiotoxicity of the produced waste remains an important issue. The most significant contributions come from plutonium and minor actinides such as ${ }^{237} \mathrm{~Np}$, ${ }^{241,243} \mathrm{Am}$, and ${ }^{245} \mathrm{Cm}$, whose half lives span from hundreds to tens of thousand of years.

A possible solution to the waste problem could derive from incineration or transmutation in subcritical reactors, like Accelerator Driven Systems (ADS), or in critical systems, like future Gen-IV fast nuclear reactors. The incineration process relies on neutron induced fission of Trans Uranic Element (TRU's,) and neutron capture on fission fragments.

Another possible way to reduce production of minor actinides is to use fissile ${ }^{233} \mathrm{U}$ in the thorium fuel cycle instead of the currently adopted ${ }^{235} \mathrm{U}$; the lower mass of the fertile ${ }^{232} \mathrm{Th}$ leads to a production of a smaller amount of actinides.

Of crucial importance for a feasibility study as well as to find the best parameters for the safe operation of such reactors, is precise knowledge of the neutron induced cross sections on a large variety of isotopes over a large energy range. Nuclides of interest span the range from the heavier minor actinides to lighter fission fragments and structural materials.

To provide these neutron induced cross section data, a neutron Time of Flight facility (n_TOF) has been built at CERN, the European Organization for Nuclear Research.

\section{THE n_TOF FACILITY}

The $\mathrm{n}$-TOF facility is driven by a $20 \mathrm{GeV} / \mathrm{C}$ proton beam from the CERN proton synchrotron (PS). A white neutron source, spanning the energy range from thermal to $300 \mathrm{MeV}$ is produced via spallation reactions when the PS proton beam strikes a $80 \times 80 \times 60 \mathrm{~cm}^{3}$ lead block. A 6-cm thick layer of light water surrounding the lead block acts both as a coolant as well as a moderator. The main characteristics of the facility are a wide energy range, low and well characterized backgrounds, excellent energy resolution (the flight path is $187.5 \mathrm{~m}$ ) and very high instantaneous neutron flux ( $310^{5}$ $\mathrm{n} / \mathrm{cm}^{2} /$ pulse with fission collimator); a crucial feature when making measurements on radioactive samples. The PS proton beam has a width of $6 \mathrm{~ns}$, and can be operated either in dedicated ( $710^{12}$ protons/pulse) or parasitic mode $\left(410^{12}\right.$ proton/pulse) with 
a duty cycle of $0.8 \mathrm{~Hz}$. Different detectors can be used in the experimental hall to measure the neutron flux [1], and capture and fission cross sections [2].

\section{THE EXPERIMENTAL SET UP}

Fission events are identified by detecting one fission fragment (FF), in a Fast Ionization Chamber (FIC). Inside the FIC, a stack of several parallel-plates are separated by $5 \mathrm{~mm}$ and surrounded by a mixture of argon and tetrafluoromethane gas $\left(90 \% \mathrm{Ar}+10 \% \mathrm{CF}_{4}\right)$ at 720 mbar pressure. There are 16 targets and 18 electrodes in total, spanning $50 \mathrm{~cm}$ in length, thus allowing a simultaneous measurement of the fission cross sections of several isotopes. Each target consisted of a stainless steel holder with a $100-\mu \mathrm{m}$ thick aluminum foil backing, and very a thin layer of target material $\left(4-450 \mu \mathrm{g} / \mathrm{cm}^{2}\right)$ deposited on both sides over a circular area $80 \mathrm{~mm}$ diameter [2].

\section{EVENT SELECTION AND DATA ANALYSIS}

An amplitude threshold was used to discriminate FF signals from background (mostly due to alpha decay of the radioactive samples) in the FIC (see figure 1).

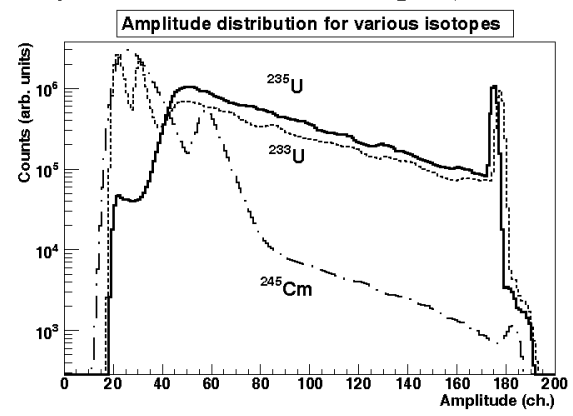

FIGURE 1: Amplitude distribution of FIC signals. The second peak for ${ }^{245} \mathrm{Cm}$ was due to the $\alpha$ decay of the nucleus, which was less pronounced for the Uranium isotopes because of their lower activity. The last peak is due to saturation. The shape of the FF distribution is a convolution of the one fragment detection and the poor energy resolution of the FIC detector.

A simple energy threshold worked well for low activity samples such as ${ }^{235} \mathrm{U}$ and ${ }^{233} \mathrm{U}$. However, for ${ }^{245} \mathrm{Cm}$ a higher threshold was needed, which cut out some low energy FF events; thus, a subsequent normalization to a known cross section was needed in this case.

\section{The neutron flux}

The neutron flux was derived from our ${ }^{235} \mathrm{U}$ measurement using the latest ENDF/BVII.0 evaluation Because of the unknown efficiency of the FIC detector, the convolution of the neutron flux and the efficiency is reported in figure 2, although the efficiency is expected to be close to $100 \%$ for the applied threshold. As expected, the 
flux shape is very similar to that measured with the capture setup, having peaks near thermal energy and $1 \mathrm{MeV}$ (the latter due to evaporation from spallation products) and an isolethargic flux shape in between.

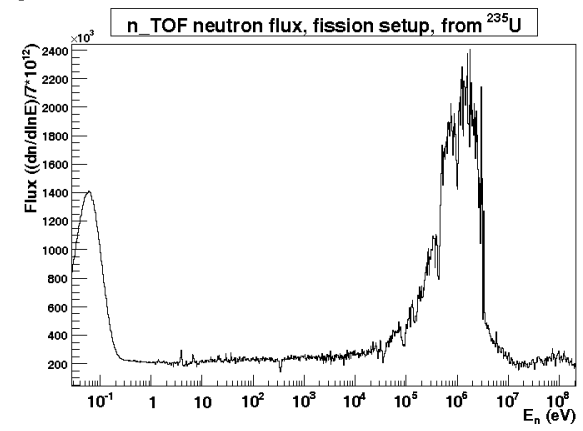

FIGURE 2:n_TOF neutron flux measured with fission collimator. The flux was calculated using the standard ${ }^{235} \mathrm{U}$ cross section taken from ENDF/B-VII.0.

\section{RESULTS}

The resultant ${ }^{233} \mathrm{U}$ fission cross section, using a threshold cutoff at channel 40 (see Fig. 1) is shown in Fig. 3. The same threshold was used in calculating the neutron flux.

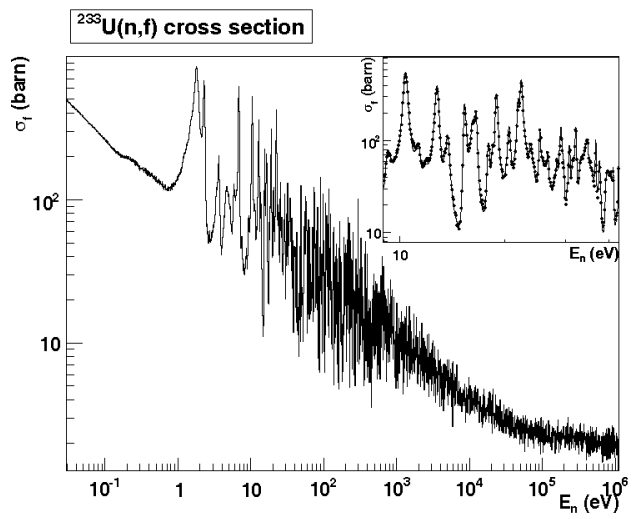

FIGURE 3: ${ }^{233} \mathrm{U}(\mathrm{n}, \mathrm{f})$ cross sections from $\mathrm{n}_{-}$TOF data (dots) and ENDF/B-VII.0 (solid curve). In general, there is good agreement between the two except that some dips in our data are more pronounced than in the evaluation, suggesting that our background may be lower than that in the measurements on which the evaluation is based.

The ${ }^{245} \mathrm{Cm}$ sample had an activity of $0.4 \mathrm{GBq}$, with alpha and spontaneous fission decay, so a simple higher energy threshold could not reject all the background. The main alpha background rejection was obtained using a threshold at channel 80. Further alpha and spontaneous fission background subtraction was accomplished using runs without neutron beam and applying the same energy threshold. Our data were normalized to previous results at thermal energy using an average weighted value of all available data [3-5]. The contribution due to the $6.6 \%$ of ${ }^{244} \mathrm{Cu}$ in the sample was subtracted. Our data are compared to the ENDF/B-VII.0 evaluation in Fig. 4. 


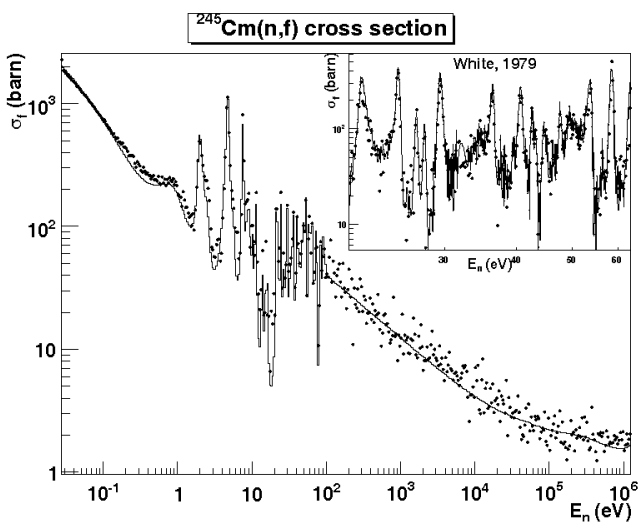

FIGURE 4: ${ }^{245} \mathrm{Cm}(\mathrm{n}, \mathrm{f})$ cross sections from $\mathrm{n} \_\mathrm{TOF}$ (dots) and ENDF/B-VII.0 (solid curve) from 0.035 $\mathrm{eV}$ to $1 \mathrm{MeV}$. In the inset, $\mathrm{n}_{-} \mathrm{TOF}$ data (dots) are compared to those from White et al. [6] (solid curve) from 20 to $60 \mathrm{eV}$.

There is a significant difference (up to $10 \%$ ) between our data and ENDF/B VI in the energy region from thermal to $20 \mathrm{eV}$. There is better agreement at higher energies, although the peak-to-valley ratio differs by about $20 \%$ for some resonances. As shown in the upper panel of figure 4, our data are in good agreement with those of White et al [6].

\section{CONCLUSIONS}

We have reported preliminary neutron induced fission cross section measurements for ${ }^{233} \mathrm{U}$ and ${ }^{245} \mathrm{Cm}$ from thermal energy to $1 \mathrm{MeV}$. Our results are, in general, in good agreement with the latest ENDF/B-VII.0 evaluation. These results demonstrate the capability to perform high precision fission cross section measurements on highly radioactive samples at the $\mathrm{n}_{-} \mathrm{TOF}$ facility. Also, the neutron flux with the fission collimator at $\mathrm{n}_{-}$TOF was determined using the well known ${ }^{235} \mathrm{U}(\mathrm{n}, \gamma)$ cross section. Detailed uncertainty estimation and resonance fitting using the R-matrix code SAMMY are in progress.

\section{REFERENCES}

1. S. Marrone et al. Nucl. Instr. and Methods. A, 517 ( 2004) 389.

2. U. Abbondanno et al., n_TOF Performance Report, CERN/INTC-O-011, INTC-(2002-037).

3. C. Browne et al., Nucl. Sci. Eng. 65 (1978) 166.

4. V. D. Gavrilov et al., Atomnaya Energiya 41 (1975) 185.

5. R. W. Benjamin et al., Nucl. Sci. Eng. 47 (1972) 203.

6. R. M. White and J. C. Browne, Nuclear Cross Sections for Technology, J.L. Fowler, C.H. Johnson, C.D. Bowman eds. (U.S. Department of Commerce, Washington 1980), NBS Special Publication 594, p.496, and R. M. White et al., Nucl. Data for Science and Technology, K.H. Böckhoff, ed. (Reidel, Dordrecht, 1983), p.218. 